metabolism of storage tissues has shown that in its response to salts the effective ions are the cations, although their effect is modified by the anions present. The inter-relations between organic acid metabolism and salt uptake of barley roots, already referred to, are not explained by the theory of anion respiration. Further, Lundegårdh's conclusion that a "very effective aeration is a drawback for intensive anion uptake" is not supported by our experimental evidence.

It is desirable to stress the importance, in absorption experiments on roots, of the metabolic status of the roots studied and their initial salt content. In so far as comparisons may be made of the results of Hoagland and Broyer ${ }^{3}$ on young barley roots and of Lundegardh on wheat roots of similar age, the indication is that the former material was so prepared that it had a much greater capacity for rapid salt accumulation than the latter, and thus some of the factors associated with salt absorption may have been more sharply defined. Items of technique assume considerable importance when general theories are proposed. Both in the barley root and potato disk experiments attempts were made to control the essential variables, biological and otherwise, and so to standardize the conditions and the absorbing system that clear-cut results emerged. This procedure, though laborious, is justified by results. Confidence in the principal conclusions is increased because they have not only been repeatedly verified over a period of years but also because they apply equally to the quite diverse systems which have been investigated. The implications of this, we believe impressive evidence, have not been adequately considered by Lundegårdh. It is, therefore, legitimate to suspend judgment concerning the reality of the anion respiration coэfficients - which purport to be characteristic of the anion species-until their reality is evident, not merely from the none too concordant sets of data from which this conception was originally derived, but also from investigations on other tissues by other investigators. It is our view that Lundegårdh's concept is not critically established.

Lundegårdh further cites in support of his theory certain measurements of electro-potentials which are thought to arise between the culture solution and the absorbing surface. The secure interpretation of these potentials would, however, require further understanding of the thermodynamic system involved. It has not been proved that the potentials do in fact have the origin suggested.

\footnotetext{
1 Lundegårdh, H., NATURE, 143, 203 (1939).

2 Published papers summarized in Trans. Far. Soc., 33, 1006 (1937). Papers on the biochemistry of salt absorption to appear in Plant
Physiology in collaboration with C. Preston and P. S. Stout. 3 Hoagland, D. R., and Broyer, T. C., Plant Physiol., 11, 471 (1936). - Prevot, P., and Steward, F. C., Plant Physiol., 11, 509 (1936).

${ }^{5}$ Lundegårdh, H., Biochem. Z., 290, 104 (1937).
}

\title{
Modern Views on Magnetism
}

\section{Conference at Strasbourg}

A CONFERENCE on magnetism was held at Strasbourg during May 21-25 under the auspices of the International Institute of Intellectual Co-operation and of the Service Central de Recherche Scientifique de France. The discussions were directed by Prof. Pierre Weiss, to whom and to whose colleagues so much of the progress in the subject is due. As well as the members of the Strasbourg laboratories, there were some thirty specialists from other institutions.

The conference brought home the contrast between the positions of the theories of paramagnetic salts on one hand, and ferromagnetism and paramagnetism in metals on the other. Paramagnetism stands in rather the same relation to quantum theory as astronomy does to Newtonian mechanics, in being the field in which results in the most detailed agreement with experiment can be obtained. This happy state of affairs is due mainly to the theoretical work of Kramers and Van Vleck, and to the experimental work carried out at Strasbourg, Leyden, Madrid and elsewhere. Reports on the present state of the subject by Foex, Cabrera and by Van Vleck revealed, it is true, a number of unexplained facts, but the general atmosphere was one of agreement between theory and experiment. The same may be said of the results on magneto-optics presented by Becquerel, Bizette, Rabinovitch and Cotton, and Ollivier.

The discovery of the magnetic cooling method, discussed in a report by Simon, has given an added importance to paramagnetism of salts and suggested new problems for investigation, for example, that of paramagnetic relaxation. A report on paramagnetic relaxation was presented by Gorter and Kronig. The question at issue is the time taken for the atomic magnets in a paramagnetic crystal to get into equilibrium when the field is switched on. For nuclear spins this time may possibly be so long as to prevent their use for reaching low temperatures. For atomic magnets, the authors quoted suggest two mechanisms by which the energy of magnetization can be dissipated. The actual magnetic interaction between the elementary magnets, small though it is, is capable of taking up some energy, and under certain conditions will absorb the energy of magnetization before this energy can be communicated to the lattice vibrations. The effect of applying a field will thus be to lower the temperature of the assembly of magnets below that of the lattice. If, however, the crystal is already in a field of more than about 2,000 gauss, this cannot happen; an elementary magnet can only come into equilibrium by giving up its energy directly to the lattice vibrations.

In metals, and especially in ferromagnetic metals, we are much further from being able to solve the equations of quantum mechanics than for paramag. netic salts. This is because we have here to do with exchange interaction between the atoms; since the electrons are continually changing places, one has to treat the whole assembly of electrons in the metal as one dynamical system, whereas a paramagnetic ion in a crystal can to a very good approximation be treated as moving in an electrostatic field. One starts from one or other of two models ; the collective electron or Bloch model, in which the electrons are 
treated as running through the lattice as a whole independently of each other, and the London-HeitlerHeisenberg model, in which the electrons are supposed to be bound to individual atoms and to move only by changing places. The relation between these models was discussed by Néel, Stoner, Mott and others, but remains by no means clear. It may, however, be of interest to give the description of a ferromagnetic metal or alloy which emerged from the discussions.

A metal such as nickel must be thought of as a mixture of ions in different states, $(3 d)^{10},(3 d)^{9}$ and perhaps higher states of ionization. The conduction electrons move freely among the ions, and number about 0.6 to 0.7 per atom. The elementary magnets are the 'holes' or vacant places in the nearly completed shells of the ions. The holes in the ions are continually moving about from ion to ion; to this motion is ascribed the large electronic specific heats observed in iron, nickel, palladium and platinum at helium temperatures. If ions with the configurations $(3 d)^{8}$ are present, then pairs of coupled spins with a moment of two Bohr magnetons will move through the crystal. The possible energy states for these holes, or pairs of holes, form bands with an energy spread of about 3 electron volts, as shown by experiments on soft X-rays (Farineau, Skinner). However, there may well be strong correlation forces which prevent more than one elementary magnet from ever being in one atom. If this is the case the correct model for a ferromagnetic material would seem to be of the Heisenberg type, modified to take account of the fact that the number of lattice points is greater than the number of magnets, a fact which allows the free motion of the magnets (holes) already referred to. An interesting first step in this direction has been taken by Stoner, who has worked out the effect of introducing a Weiss intramolecular field into the collective electron treatment.

For nickel and nickel-rich alloys the total number of positive holes in the $d$ shells is equal to the saturation moment in Bohr magnetons ; the report of Néel showed very clearly that this cannot be the case for iron. Here the ions must be present in a large number of different states of ionization, and only some of them give a positive exchange integral and thus a contribution to the ferro-magnetism.

There were interesting discussions on what happens to the orbital moment in ferromagnetic materials, evidence being drawn from the gyromagnetic effect (Sucksmith, Barnett) and from the saturation moments of alloys (Forrer). From the theoretical point of view this problem remains quite obscure.

Becker, Sucksmith and Gerlach gave results bearing on the magnetization curves of ferromagnetic materials. A large number of facts seem to indicate that a small piece of material has a high coercive force. According to Becker, a magnetized material contains nuclei at which demagnetization can start, and such nuclei would not be present in a small specimen; but no explanation could be given of the nature of these nuclei.

The diamagnetism of aromatic crystals was discussed by Krishnan, in particular their magnetic anisotropy. As pointed out by F. London, the 'orbit' responsible for the diamagnetism in naphthalene, anthracene and so on must extend over the whole molecule.

\section{Work of the Discovery Committee}

\section{Fifth Commission of the R.R.S. Discovery II}

$\mathrm{T}$ HE Discovery Committee's vessel R.R.S. Discovery $I I$, built in 1929 for oceanographical research in the Southern Ocean, returned to London from her fifth voyage on May 9. Leaving Fingland in October 1937, she spent the southern summer circumnavigating the antarctic continent, making observations along the northern edge of the pack-ice south of the Indian, Pacific and Atlantic Oceans, and running lines of stations across the antarctic, subantaretic and subtropical regions as she came northwards to Australia, New Zealand and the Falkland Islands for fuel.

In the subtropical and subantarctic regions a full station was worked every day : temperatures were measured and water samples taken at a series of depths between the surface and the bottom, and nets were fished to depths of at least 1,000 metres. In the antarctic zone extra nets were fished to obtain a more complete picture of the distribution of the small prawn-like krill (Euphausia superba) on which the whales feed, and a constant look-out was kept for whales. Near the ice-edge, and in regions where the krill are known to breed, the work was still more intensive.

A qualitative picture of the water circulation in the Southern Ocean and the distribution of factors that influence plant and animal growth had been obtained on previous voyages, and although this latest voyage extended the work whenever possible, its main object was the collection of data for comparison with previous observations, particularly in regard to the contrast between summer and winter conditions. The circumpolar voyage was completed early in May 1938, and the remainder of the commission was devoted to the systematic survey, month by month, of a limited section of the Southern Ocean. Such repeated observations are of great assistance in the elucidation of oceanographical problems, and it is noteworthy that the International Council for the Exploration of the Sea is planning a similar though more concentrated survey of the Faroe - Shetland region.

The area chosen was the antarctic sector between the Greenwich meridian and $20^{\circ} \mathrm{E}$. : this afforded a good opportunity for the examination of typical antarctic, subantarctic and subtropical conditions, and at the same time it allowed a continuous examination of the terminal region of the. Weddell Sea current, a matter of great importance, since the Weddell Sea has been found to be by far the greatest centre of production of krill.

After refitting in Simonstown, the Discovery II steamed southwards along the Greenwich meridian as far as the ice-edge, and after a short cruise away from the ice to a point some 300 miles to the northward in $10^{\circ} \mathrm{E}$., she returned northwards along $20^{\circ} \mathrm{E}$. Between July 1, 1938, and Murch 19, 1939, this voyage, south and north, was made seven times, and 